\title{
ESTRUTURADO DE MIX DE POLPAS DE UMBU E JAMELÃO
}

\author{
Angélica Maria Oliveira Mascarenhas ${ }^{1}$; Ernesto Acosta Martinez ${ }^{2}$; Geany Peruch \\ Camilloto $^{3}$; Larissa Silva Seixas ${ }^{4}$; Sílvia Maria Almeida de Souza ${ }^{5}$ \\ 1. Bolsista PIBITI/CNPq, Graduando em Engenharia de Alimentos, Universidade Estadual de Feira de Santana (UEFS), e- \\ mail: angelica.gel07@gmail.com \\ 2. Orientador, Departamento de Tecnologia, UEFS, e-mail: ernesto.amartinez@yahoo.com.br \\ 3. Participante do projeto, Departamento de Tecnologia, UEFS, e-mail: geanyperuch@ yahoo.com.br \\ 4. Voluntária, Graduando em Engenharia de Alimentos, UEFS, e-mail: larissa.sseixas@ hotmail.br \\ 5. Participante do projeto, Departamento de Tecnologia, UEFS, e-mail: $\underline{\text { ss_almeida@ yahoo.com.br }}$
}

PALAVRAS-CHAVE: Estruturado; frutas; hidrocolóides

\section{INTRODUÇÃO}

Dentre as técnicas de processamento, a estruturação de polpa de frutas representa uma inovação na área de alimentos, com resultados bastante promissores (CARVALHO et al., 2008). Alimento estruturado ou "designed food" ou "engineered food", são delineados de acordo com um planejamento, geralmente empregando-se matérias-primas de baixo custo, oriundas de frutas que se encontram fora de classificação para comercialização in natura ou excedentes de produção durante o período de safra, em muitos casos, utilizando-se hidrocolóides (FIZMAN, 1992 apud GRIZOTTO et al., 2005). Os hidrocolóides como alginato, pectina e gelatina, irão atuar como agentes de união, facilitando o corte e retendo umidade, contribuindo para a melhoria da textura (GRIZOTTO et al., 2005).

O presente trabalho tem como objetivos estudar o efeito da massa de pectina, alginato e gelatina na formulação do estruturado a partir de um mix de polpa de umbu e jamelão: um produto inovador para ser comercializado pelos agricultores aumentando assim sua renda.

\section{MATERIAL E MÉTODOS}

Os frutos foram sanitizados com solução clorada e selecionados, o umbu foi deempolpado em despolpadeira semi-industrial Itametal 165 e o jamelão, manualmente. A concentração da polpa de umbu foi realizada em rotoevaporador Fisatam 802 nas condições de temperatura do banho de $50-55^{\circ} \mathrm{C}$, rotação de $120 \pm 5 \mathrm{rpm}$ e vácuo de $700 \mathrm{mmHg}$ (GRIZOTTO et al., 2005). A seguir, a polpa concentrada será congelada. A polpa de jamelão não foi concentrada.

Nas formulações dos estruturados foram utilizados $30 \mathrm{~g}$ de polpa concentrada $(29,4 \mathrm{~g}$ de umbu e $0,6 \mathrm{~g}$ de jamelão), glicerina ( $10 \%$ em relação à polpa) aquecendo a $60^{\circ} \mathrm{C}$ por 5 min sob agitação e açúcar refinado suficiente para elevar o teor de sólidos solúveis até $50^{\circ}$ Brix. Posteriormente, foi adicionada uma mistura seca de alginato, pectina e gelatina, agitando por mais $5 \mathrm{~min}$. Foi adicionado uma suspensão de $0,48 \mathrm{~g}$ de fosfato de cálcio em $2 \mathrm{~mL}$ de água destilada, agitando por mais 5 minutos. A moldagem das frutas estruturadas $(50 \mathrm{~g})$ foram realizadas em placas de Petri as quais foram mantidas sob refrigeração a $10^{\circ} \mathrm{C}$ durante $24 \mathrm{~h}$ para completar a geleificação (GRIZOTTO et al., 2005). Depois de retirados dos moldes, os estruturados foram submetidos à secagem a $45^{\circ} \mathrm{C}$ durante $5 \mathrm{~h}$ a fim de reduzir a água livre do produto e minimizar a adesividade excessiva na superfície das frutas estruturadas (CARVALHO et al., 2008). Os ensaios foram realizados segundo planejamento fatorial $2^{3}$ usando pectina ( 0,92 a 2,92 g), alginato $(0,30$ a $0,90 \mathrm{~g})$ e gelatina ( 2 a $4 \mathrm{~g})$ segundo Rodrigues e Iemma (2009).

Nas avaliações físico-química foram determinados o teor de sólidos solúveis por refratômetro, atividade de água, $\mathrm{pH}$, cinzas, proteínas, acidez total, carboidrato e vitamina $\mathrm{C}$ total das polpas in-natura e concentrada. Foram realizadas análises de firmeza, atividade de água, umidade, sólidos solúveis e totais dos estruturados segundo metodologia de Grizotto et 
al. (2005). A análise estatística dos dados foi realizada através da análise de variância ANOVA e da metodologia da superfície de resposta empregando-se o programa Statistica 7.0.

\section{RESULTADOS E DISCUSSÃO}

$\mathrm{Na}$ Tabela 01 são apresentadas as características físico-químicas das polpas de umbu in natura e concentrada e da polpa de jamelão.

Tabela 01. Características físico-química das polpas de umbu in natura e concentrada e de jamelão.

\begin{tabular}{lccc}
\multicolumn{1}{c}{ Propriedade } & $\begin{array}{c}\text { Polpa de umbu in } \\
\text { natura }\end{array}$ & $\begin{array}{c}\text { Polpa de umbu } \\
\text { concentrada }\end{array}$ & Jamelão \\
\hline Acidez titulável (\%) & $26,82 \pm 0,05$ & $36,59 \pm 0,04$ & $10,34 \pm 0,08$ \\
Sólidos solúveis $\left({ }^{\circ}\right.$ Brix) & $11 \pm 0,00$ & $15 \pm 0,00$ & $12 \pm 0,00$ \\
Carboidratos redutores (\%) & $1,69 \pm 0,15$ & $3,38 \pm 0,10$ & $6,93 \pm 0,21$ \\
Carboidratos totais (\%) & $5,88 \pm 1,30$ & $8,5 \pm 0,0$ & $16,77 \pm 0,15$ \\
Cinzas (\%) & $0,64 \pm 0,02$ & $0,90 \pm 0,05$ & $0,55 \pm 0,00$ \\
pH & $2,41 \pm 0,00$ & $2,43 \pm 0,00$ & $3,44 \pm 0,00$ \\
Proteína (\%) & $1,93 \pm 0,39$ & $1,94 \pm 0,31$ & $0,55 \pm 0,55$ \\
Vitamina C (\%) & $2,33 \pm 0,01$ & $1,72 \pm 0,14$ & - \\
Atividade de água & $0,985 \pm 0,00$ & $0,983 \pm 0,00$ & $0,984 \pm 0,00$ \\
\hline
\end{tabular}

Verificou-se na polpa in natura e após concentrada um aumento significativo de aproximadamente $37 \%$ na propriedade acidez; os teores de sólidos solúveis e de açúcares totais aumentaram em 1,36 e 1,6 vez respectivamente depois da concentração da polpa. Entretanto, foi constatado um aumento maior na concentração de açúcares redutores $(2,1$ vezes). Com relação ao teor de cinzas, verificou-se um aumento de $0,64 \%$ até $0,9 \%$ após a concentração da polpa (Tabela 1). O teor de cinzas depende do tipo de solo e da composição do mesmo (BURVALL, 1997; KHAN et al., 2014).

$\mathrm{O}$ processo de concentração não teve influencia sobre o valor de $\mathrm{pH}$ da polpa tendo valores $(2,4)$ similares. Após a concentração da polpa de umbu, pode-se constatar uma diminuição da concentração de vitamina $C(26,18 \%)$. Os teores de vitamina $C$ da polpa in natura e concentrada são consideravelmente inferiores aos reportados na literatura. Isso pode ser devido a uma degradação mais intensa da vitamina com o período de estocagem, o contato com o oxigênio atmosférico ou com a ação da luz (OYETADE et al., 2012). A polpa de jamelão contém maior concentração de carboidrato total e redutor que a polpa concentrada de umbu. As características dos estruturados obtidos em cada formulação do planejamento experimental são apresentadas na Tabela 02.

\begin{tabular}{cccccccc}
\multicolumn{2}{c}{ Tabela 02. Caracterização físico-química das formulações de estruturado de mix de } \\
polpas de umbu com jamelão. \\
$\begin{array}{c}\text { Formu- } \\
\text { lações }\end{array}$ & $\mathrm{pH}$ & $\begin{array}{c}\text { Sólidos } \\
\text { solúveis } \\
\left({ }^{0} \mathrm{Brix}\right)\end{array}$ & $\begin{array}{c}\text { Peso seco } \\
(\%)\end{array}$ & $\begin{array}{c}\text { Perda de } \\
\text { umidade } \\
(\mathrm{g})\end{array}$ & $\begin{array}{c}\text { Atividade de } \\
\text { água }\end{array}$ & Firmeza (g) & $\begin{array}{c}\text { Elastici- } \\
\text { dade }(\%)\end{array}$ \\
\hline 1 & $3,30 \pm 0,0$ & $58 \pm 0,0$ & $13,95 \pm 0,04$ & $0,81 \pm 0,00$ & $0,71 \pm 0,01$ & $83,76 \pm 2,01$ & $49,96 \pm 0,9$ \\
2 & $3,38 \pm 0,0$ & $58 \pm 0,0$ & $13,71 \pm 0,03$ & $4,44 \pm 0,15$ & $0,70 \pm 0,01$ & $92,54 \pm 4,02$ & $42,78 \pm 2,1$ \\
3 & $3,25 \pm 0,0$ & $58 \pm 0,0$ & $12,65 \pm 0,05$ & $4,43 \pm 0,10$ & $0,67 \pm 0,00$ & $125,43 \pm 3,0$ & $41,12 \pm 2,0$ \\
4 & $3,13 \pm 0,0$ & $58 \pm 0,0$ & $10,85 \pm 0,02$ & $0,66 \pm 0,00$ & $0,68 \pm 0,01$ & $72,55 \pm 4,07$ & $41,36 \pm 1,2$ \\
\hline
\end{tabular}




\begin{tabular}{clllllll}
\hline 5 & $3,26 \pm 0,0$ & $58 \pm 0,0$ & $8,27 \pm 0,01$ & $3,69 \pm 0,18$ & $0,60 \pm 0,03$ & $63,33 \pm 5,05$ & $38,52 \pm 2,1$ \\
6 & $3,02 \pm 0,0$ & $58 \pm 0,0$ & $8,45 \pm 0,02$ & $3,75 \pm 0,05$ & $0,63 \pm 0,01$ & $45,52 \pm 6,42$ & $34,03 \pm 2,3$ \\
7 & $3,25 \pm 0,0$ & $58 \pm 0,0$ & $9,84 \pm 0,03$ & $4,22 \pm 0,06$ & $0,65 \pm 0,01$ & $37,66 \pm 1,02$ & $45,78 \pm 1,1$ \\
8 & $3,15 \pm 0,0$ & $58 \pm 0,0$ & $9,65 \pm 0,01$ & $0,88 \pm 0,00$ & $0,69 \pm 0,01$ & $68,28 \pm 8,02$ & $37,59 \pm 2,0$ \\
9 & $3,21 \pm 0,0$ & $58 \pm 0,0$ & $9,06 \pm 0,04$ & $3,84 \pm 0,25$ & $0,65 \pm 0,01$ & $55,30 \pm 6,04$ & $40,80 \pm 1,5$ \\
10 & $3,18 \pm 0,0$ & $58 \pm 0,0$ & $9,51 \pm 0,05$ & $3,92 \pm 0,02$ & $0,65 \pm 0,01$ & $54,91 \pm 3,05$ & $41,62 \pm 1,1$ \\
11 & $3,20 \pm 0,0$ & $58 \pm 0,0$ & $11,48 \pm 0,02$ & $4,05 \pm 0,06$ & $0,67 \pm 0,00$ & $57,19 \pm 2,51$ & $42,37 \pm 0,9$ \\
\hline
\end{tabular}

$\mathrm{Na}$ análise estatística da resposta perda de umidade (PU, \%) do estruturado do mix de polpas de umbu e jamelão foi verificado que não houve efeito significativo das massas de pectina, alginato e gelatina utilizadas. A análise aponta que os efeitos da pectina e da interação entre pectina e alginato são significativas ao nível de $95 \%$ de confiança com um coeficiente de determinação $\left(\mathrm{R}^{2}\right)$ de 0,9571. A análise estatística dos resultados obtidos demonstra que não existe, dentro da região experimental estudada, um efeito acentuado de curvatura. Logo, o modelo de primeira ordem é mais adequado para a resposta Aw e o efeito da falta de ajuste não foi significativo.

De acordo com as superfícies de resposta (Figura 01), no gráfico A pode-se constatar que maiores valores de Aw no estruturado de mix de polpas de umbu e jamelão $(0,700)$ são obtidos nas condições de menores massas de pectina $(1,75 \mathrm{~g})$. No gráfico B pode-se constatar que maiores valores de PU no estruturado de mix de polpas de umbu e jamelão $(4 \mathrm{~g})$ são obtidos nas condições de maiores massas de alginato $(0,45 \mathrm{~g})$ e menores de pectina $(0,75 \mathrm{~g})$. No gráfico $\mathrm{C}$ pode-se constatar que maiores valores de firmeza no estruturado de mix de polpas de umbu e jamelão (80 g) são obtidos nas condições de menores massas de pectina $(1,75 \mathrm{~g})$ independentemente da massa de alginato. No gráfico D pode-se constatar que maiores valores de elasticidade do estruturado de mix de polpas de umbu e jamelão (46\%) são obtidos nas condições de maiores massas de gelatina $(2,50 \mathrm{~g})$ e pectina $(1,75 \mathrm{~g})$. $\mathrm{O}$ uso de menores massas de pectina $(0,75 \mathrm{~g})$ e gelatina $(1,5 \mathrm{~g})$ facilita a obtenção de estruturados com menores valores de elasticidade $(35,9 \%)$. Nas condições de maior massa de pectina $(1,75 \mathrm{~g})$ independentemente das massas de gelatina usadas constata-se a obtenção de estruturados com valores de elasticidade de aproximadamente $42 \mathrm{~g}$. Com o uso de maior massa de gelatina $(2,5$ g) o aumento da massa de pectina de 0,75 para 1,75 g produziu um aumento de $8 \%$ na elasticidade do estruturado.

\section{CONSIDERAÇÕES FINAIS}

É possível obter estruturados de mix de polpas de umbu e jamelão. Na análise estatística foi verificado que não houve efeito significativo das massas de pectina, alginato e gelatina utilizadas em relação perda de umidade (PU, \%) do estruturado do mix de polpas de umbu e jamelão. Maiores valores de atividade de água no estruturado $(0,700)$ são obtidos nas condições de menores massas de pectina $(1,75 \mathrm{~g})$; e que maiores valores de perda de umidade no estruturado $(4,0 \mathrm{~g})$ são obtidos nas condições de maiores massas de alginato $(0,45 \mathrm{~g})$ e menores de pectina $(0,75 \mathrm{~g})$. $\mathrm{O}$ uso de menores massas de pectina $(1,75 \mathrm{~g})$ e gelatina $(1,5 \mathrm{~g})$ facilita a obtenção de estruturados com menor elasticidade $(35,9 \%)$. 

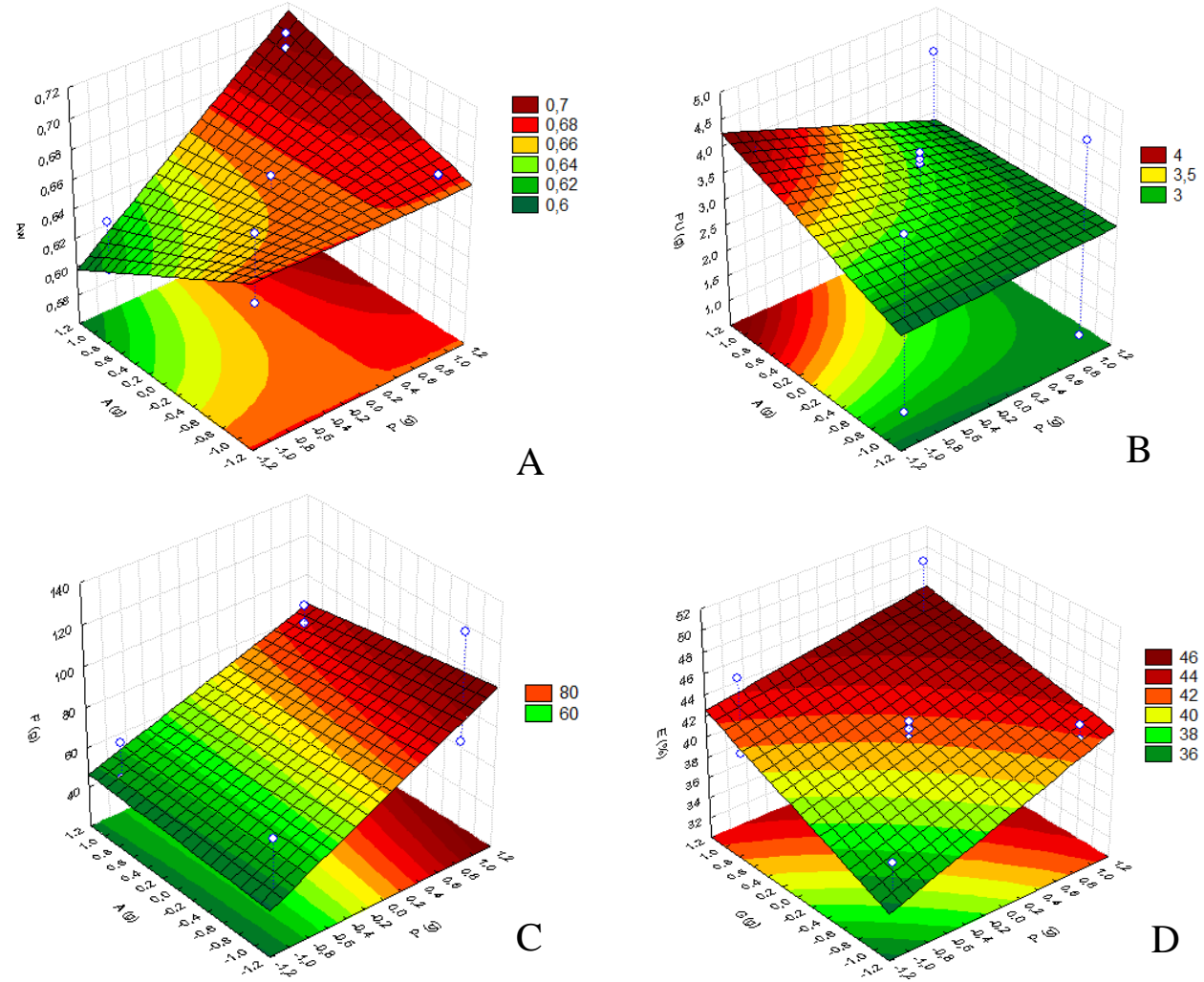

Figura 01. Superfície de resposta descrita pelo modelo proposto, que representa: em A atividade de água (Aw); em B perda de umidade (PU, g); em C firmeza do estruturado de mix de polpas de umbu e jamelão; em D a elasticidade $(\mathrm{E}, \%)$ do estruturado de mix de polpas de umbu e jamelão em função da massa de pectina $(\mathrm{P})$ e de gelatina $(\mathrm{G})$.

\section{REFERÊNCIAS}

BURVALL, J. Influence of harvest time and soil type on fuel quality in reed canary grass (Phalaris arundinacea L.). Biomass and Bioenergy, v.12, n.3, p.149-154, 1997.

CARVAlHO, A. V.; MATTIETTO, R. A.; VASCONCELOS, M. A. M. Aproveitamento da Casca do Bacuri para Fabricação de um Novo Produto. Comunicado Técnico 209, Embrapa. Belém- PA, Setembro, 2008.

GRIZOTTO, R. K.; BRUNS, R. E.; AGUIRRE, J. M.; BATISTA, G. Otimização via metodologia de superfície de respostas dos parâmetros tecnológicos para produção de fruta estruturada e desidratada a partir de polpa concentrada de mamão. Ciência e Tecnologia de Alimentos, v. 25, n.1, p. 158-164. Campinas, jan/ mar, 2005.

KHAN, A. A.; SAJID, M.; RAD, A. Tomato fruit quality as affected by different sources of phosphorus. Pakistan Journal of Nutrition, v.13, n.12, p.692-699, 2014.

ROBRIGUES, M. I.; IEMMA, A. F. Planejamento de experimentos e otimização de processos. 2. ed. Campinas - SP: Casa do Espírito Amigo Fraternidade Fé e Amor, 2009.

OYETADE, O. A.; OYELEKE, G. O.; ADEGOKE, B. M.; AKINTUNDE, A. O. Stability Studies on Ascorbic Acid (Vitamin C) From Different Sources. IOSR Journal of Applied Chemistry, v.2, n.4, p.20-24, 2012. 